\title{
DECONSTRUCCIÓN SOCIAL DE NEGRAS Y NEGROS EN CUBA. CONTEXTO ACTUAL
}

\section{SOCIAL DECONSTRUCTION OF BLACK WOMEN AND BLACK MEN IN CUBA. CURRENT CONTEXT}

Sandraliz Rafoso Pomar. Centro de Desarrollo Local y Comunitario (CEDEL), Cuba. sandraliz@cedel.cu

\section{RESUMEN}

El trabajo ofrece elementos teóricos-conceptuales simplificados, sobre la construcción social, deconstrucción, racismo, casas comunitarias, ambiente digital, medios de comunicación masiva. A su vez se tiene en cuenta cómo estas categorías, discursos y lecturas sociales, transforman la interacción de sujeto a sujeto; en un contexto, donde poco a poco se logra visibilizar la problemática racial, en espacios formales e informales. En este sentido, el presente trabajo tiene como objetivo reflexionar acerca de cómo la construcción social, los estereotipos e imaginarios populares han afectado la imagen o la representación social de negras y negros. Se presenta la deconstrucción social desde cualquier frente académico, cultural, social e investigativo, y de cómo ha ido presentando resultados impresionantes, en un escenario que todavía los debates públicos sobre el tema racial no son suficientes. La metodología utilizada fue la cualitativa. Se realizó la consulta de fuentes documentales y no documentales, punto de partida para el desarrollo de esta investigación. La técnica metodológica utilizada fue el análisis documental que permitió conocer el estado del arte del tema, así como las posturas de diferentes autores. Se presentan conclusiones y bibliografía.

PALABRAS CLAVE: construcción social; deconstrucción social; racismo; medios de comunicación; Cuba; ambiente digital.

\section{ABSTRACT}

The paper offers simplified theoretical-conceptual elements, on social construction, deconstruction, racism, community houses, digital environment, mass media. At the same time, it is taken into account how these categories, speeches and social readings transform the interaction from subject to subject; in a context, where racial problem is gradually becoming visible, in formal and informal spaces. In this sense, the present work aims to reflect on how social construction, stereotypes and popular imaginary have affected the image or social representation of black women and black men. Social deconstruction is presented from any academic, cultural, social and research front, and how it has been presenting impressive results, in a scenario in which public debates on the racial issue still are not enough. The methodology used was qualitative. The consultation of documentary and non-documentary sources, starting point for the development of this research was carried out. The methodological technique used was the documentary analysis that allowed to know the state of the art of the subject, as well as the positions of different authors. Conclusions and bibliography are presented. 
KEYWORDS: social construction; social deconstruction; racism; media; Cuba; digital environment.

\section{Cómo citar el artículo:}

Rafoso Pomar, S. (2019). Deconstrucción social de negras y negros en cuba. Contexto actual. Revista de Ciencias de la Comunicación e Información, 24(1), 29-40. doi: http://doi.org/10.35742/rcci.2019.24(1).29-40

\section{INTRODUCCIÓN}

El lunes 16 de julio comenzó en La Habana la Segunda Escuela Internacional de Posgrado, "Más Allá del Decenio de los Pueblos Afrodescendientes". Un escenario, donde estudiantes, profesores, investigadores, escritores y personalidades del mundo académico y de la cultura, con sapiensa, inteligencia y activismo, luchan en contra de la discriminación racial, injusticia y visibilidad de la problemática racial en contexto nacional e internacional. El acto de inicio del posgrado, fue un homenaje histórico-simbólico, a Mariana Grajales. Esa mañana, testigos de un momento inolvidable, se inició el debate, confrontación y discrepancias, sobre la construcción de la realidad, desconstrucción y aquellos imaginarios sociales, positivos o negativos en la sociedad actual.

El firme propósito de cada uno de los participantes en la escuela, era intercambiar, hablar de sus experiencias, y volcar las dudas y las inconformidades, en un espacio listo ideológicamente y cognitivamente, para comprender más la humanidad, las huellas de la historia y el curso de ella; porque en el contexto actual las situaciones de la vida van más rápido de lo que a veces se piensa. El mundo de hoy, es diferente al de ayer.

Primero que todo, es indispensable abrir de par en par la cuestión del término "color" referido a las características de las gentes. La idea misma de "color" en esa relación es un constructo mental. Si se dice que hay "colores" políticos ("rojos", "negros", "blancos"), todo el mundo está, presumiblemente, dispuesto a pensarlo como una metáfora. Pero, curiosamente, no ocurre así cuando se dice que alguien es de "raza blanca", o "negra", "india", "piel roja" o "amarilla". Y, más curioso aún, pocos piensan espontáneamente que se requiere una total deformación de la vista para admitir que "blanco" (o "amarillo" o "rojo") pueda ser un color de piel sano. (Quijano, 2017, p. 21).

Por tanto, el objetivo de este trabajo es reflexionar acerca de cómo la construcción social, los estereotipos e imaginarios populares han afectado la imagen o la representación del negro; y de cómo la deconstrucción social del negro, desde cualquier frente académico, cultural, social e investigativo, ha ido reivindicando la figura de este; Así, pues, aparecen las investigaciones cualitativas o cuantitativas que representan la escenificación del tejido social en modificación.

\section{OBJETIVO}

Reflexionar acerca de cómo la construcción social, los estereotipos e imaginarios populares han afectado la imagen o la representación social de negras y negros. Se presenta la deconstrucción social desde cualquier frente académico, cultural, social e investigativo, y de cómo ha ido presentando resultados impresionantes, en un escenario que todavía los debates públicos sobre el tema racial no son suficientes. 


\section{METODOLOGÍA}

La metodología utilizada fue la cualitativa. Se realizó la consulta de fuentes documentales y no documentales, punto de partida para el desarrollo de esta investigación. La técnica metodológica utilizada fue el análisis documental que permitió conocer el estado del arte del tema, así como las posturas de diferentes autores.

\section{CONSTRUCCIÓN SOCIAL DE LA REALIDAD Y DECONSTRUCCIÓN}

Lo que se reconoce como "la realidad" es una noción que se construye socialmente. Una noción propia de qué es lo real, se construye según los contextos sociales específicos; y, lo que es más interesante aún, cómo esa "realidad" puede a su vez desaparecer para un individuo o para una colectividad entera. (Berguer, Peter y Luckmann, Thomas, 2006, pp. 11-13).

Las nociones sobre qué es "lo real" varían entre los grupos humanos pertenecientes a diversas culturas y sociedades, sin que se pueda escoger entre tantas, cuál es la exacta o verdadera, ya que todas corresponden a un contexto que ha creado su sistema de valores y su cosmogonía, de acuerdo a una evolución histórica y social. Definir la "realidad" como una cualidad propia de los fenómenos que reconocemos como independientes de nuestra propia voluntad y definir el conocimiento como la certidumbre de que los fenómenos son reales y de que poseen características específicas. El interés sociológico en materia de "realidad 1" y "conocimiento" se justifica así inicialmente por el hecho de su relatividad social. (Álvarez, 2014, p. 45).

La teoría de Berguer y Luckmann aborda las funciones manifiestas o latentes de esa realidad construida socialmente. Es decir, hasta qué punto el fenómeno se da libremente sin la acción directa de grupos o instituciones, o a qué nivel este proceso es dirigido conscientemente en función de preservar los valores que fundamentan la ideología y la cultura dominante. Pero la realidad social está formada no sólo por lo que los teóricos y políticos acuñan como sistema de ideas legítimo, ya que el conocimiento se construye sobre todo en las calles y en la vida cotidiana. (Álvarez, 2014, p. 45).

Desde la célula familiar se generan prejuicios, costumbres, tradiciones, formas de ser y hacer, en sintonía o contradicción con el marco social o con lo que se percibe como una realidad óptica.

Por eso, "La sociedad es un producto humano. La sociedad es una realidad objetiva. El hombre es un producto social." (Berger y Luckmann, 2006, p. 82).

En este sentido, el hombre ha ido construyendo y organizándose, con sus subjetividades, normas, políticas, creencias y actitudes; que son visibles, en acciones; y así se observa cómo se va conformando esas categorías sociales, donde se establece y se personaliza a cada ser humano.

\footnotetext{
${ }^{1}$ Como planteara Hegel: "La realidad es, a la vez, algo dado y algo transformado, algo inmediato y algo mediato. O dicho en otra forma, la realidad tiene un modo de manifestarse siempre determinado, es así o es asá. Pero al mismo tiempo no es una apariencia estática: es producto de la negatividad, de la capacidad transformadora de la esencia" (citado en Rojo, 2009, p. 31).
} 
En el Plan de Acción de Programa del País, en la Parte II, Análisis de la Situación, "Uno de los principales desafíos para el logro de una cultura de la equidad es la permanencia de estereotipos de género en la cultura y en los imaginarios socialmente compartidos". (Gobierno de la República de Cuba- UNFPA, 2013, p. 4).

\subsection{DECONSTRUCCIÓN}

Cada día convencerse de la vulnerabilidad a la que cada sujeto está expuesto en una sociedad por los constructos estereotipados, es como una lucha constante entre querer ilustrar un olio con los colores primarios, o mostrar la gama de colores, después de ser manipulados y acomodados según la opinión subjetiva de algunos; es así como se genera una construcción social del medio, donde las cosas toman un rumbo, según los imaginarios sociales existentes.

La importancia de los imaginarios, a decir de De la Iglesia, radica en "su fuerza valorativa, ya que operan como guía en los procesos de socialización, determinando la construcción social de la realidad, la autorepresentación que la misma tiene de sí y la identidad y pertenencia modeladores de la subjetividad. (De la Iglesia, 2002).

La tesis de maestría del profesor Berto Esilio Martínez, la cual se titula Territorios de identificación: una poética de la resistencia de la gente en Turbo (2014). Esta se sitúa en la línea Pedagogía y Diversidad Cultural de Maestría en Educación. El autor parte afirmando que su trabajo es el desafío de: articular críticamente los anclajes de las identificaciones de la gente negra en Colombia para (...) descontruir imaginarios racializados y coloniales que aún perviven en las epistemologías y ontologías hegemónicas/eurocéntricas en nuestra sociedad. (Martínez, 2014).

Los esfuerzos deben dirigirse a resimbolizar los valores negativos y no reconocidos que los sistemas racista y patriarcal en su compleja relación han invisibilizado. La persistencia de conductas discriminatorias en diferentes ámbitos, unido a la permanencia de estereotipos y prejuicios racistas en nuestra sociedad, demanda una mirada crítica y constructiva de la realidad, que nos permita articular acciones certeras si queremos aniquilar un mal social que agoniza pero no muere. (Almeida, 2010, p. 29).

Es esencial decontruir esos estereotipos en Cuba y representar la verdadera historia de lo que fue. Walterio Carbonell en su libro, "Cómo surgió la Cultura Nacional" publicado por la Biblioteca Nacional José Martí en 2005 desconstruye la imagen de figuras enaltecidas en la historia de Cuba:

Hay que esclarecer el siglo XIX esclavista, porque es precisamente durante este siglo que la ociosidad es más elocuente. La burguesía tenía sus historiadores, sus periodistas, sus profesores que escribían fábulas heroicas sobre ella para que el pueblo las tomara por realidades y justificara su dominación. Es por todas estas razones que el siglo XIX necesita revisión. Dioses de barro superviven como una realidad en la conciencia de nuestro pueblo revolucionario. Figuras oscuras, esclavistas de la peor especie, como Arango y Parreño; esclavistas atormentados como José Antonio Saco y Luz Caballero, enemigos de las revoluciones y de la convivencia democrática, han sido elevados a la categoría de dioses nacionales por los historiadores, profesores y políticos burgueses. La Revolución no puede tener por dioses nacionales a estos hombres, los mismos hombres que fueron 
elevados por la burguesía a la categoría de dioses nacionales. (Carbonell, 2005, pp. 21-22).

La deconstrucción de la historiografía ha sido una ardua labor encabezada por los intelectuales que se han comprometido con el tema racial $y$, a su vez, ha significado una estrategia para combatir el racismo y alcanzar el reconocimiento del rol fundamental que desempeñaron negros y mestizos en la construcción de la nación cubana. (Casanova, 2014, p. 93).

Trabajar desde la base social más invisible de forma piramidal y horizontal, hasta la base jerárquica social, más alejada de esta realidad, hace ver, que aunque se quiera hacer mucho, cuando no se toca el fondo y no se tiene conciencia del tema; entonces, es difícil decir que se quiere decontruir algo, más bien se adorna de cortinas conceptuales y de capas oxidadas que van desprendiéndose poco a poco, desde lo local a lo nacional.

\section{ALGUNAS PRECISIONES Y OPINIONES SOBRE EL RACISMO}

El término racismo tiene, en sentido estricto, un origen y contenido bien definidos... El término surge a mediados del presente siglo comúnmente asociado, de forma peyorativa, a las doctrinas y la praxis del régimen nacionalsocialista alemán relativas a la superioridad de unas razas sobre las demás (Colino, 2009).

"La construcción del racismo, no es pues, el resultado de elucubraciones disparatadas de ignorantes. Por lo contrario, participaron en su creación algunas de las mentes más brillantes de Europa". (Duncan, s.a., p. 6).

Por eso "Entiéndase al Racismo como una condición en la que un humano desprecia a otro por alguna característica física en particular que la diferencia de quien es en mayoría se considera "Standard". (Definición de racismo, 2015).

Escritores, oradores, pensadores, críticos e investigadores, de diferentes regiones, opinan en sus libros, artículos, ponencias, investigaciones, discursos y memorias.

Uno de ellos, es la figura de Malcolm X, donde expresa que "no es el americano blanco el racista. Es la atmósfera política, social y económica lo que fomenta el racismo". (Malcolm X..., 1991, p. 131).

A su vez, Bourdieu, opina que "El racismo se alimenta de una supuesta creencia de la existencia de razas superiores, que "naturalmente", tendrían mayor capacidad de adaptación de dominación, mientras que otros serían trascendentalmente inferiores". (Bourdieu, 2000).

Oquendo se pronuncia, que "No cabe dudas de que el racismo es la herencia más ominosa de la trata esclavista". (Oquendo Barrios, 2006, p. 11).

Otras opiniones puntuales es la de Césaire citado en Grosfoguel, opina que

el racismo no era una superestructura/epifenómeno sino un rasgo constitutivo del sistema capitalista. El racismo a era la lógica que organizaba a las poblaciones del mundo en la división internacional del trabajo de centros y periferias, la cual generaba la consiguiente acumulación de capital a escala mundial que se superponía a la jerarquía racial de europeos versus no europeos respecto a la cual 
estos últimos constituían la mano de obra barata producida políticamente por medias violentos y coercitivos. (Grosfoguel, 2006, p. 149).

Stuart que puntualiza que "Debemos comenzar entendiendo que la esclavitud (como respuesta a condiciones y necesidades históricas) generó una forma particular de racismo". (Stuart, 2013, p. 267).

Por último, León reconoce que

el racismo puede aparecer como un elemento estratégico de la colonización para la manipulación de otros y la única salida para poder ideologizar en masa a la sociedad, a través de la descalificación y la utilización del concepto negro como un término peyorativo. Es necesario mencionar que esta es la huella dolosa que dejó el colonialismo cuando se permite invalidar la existencia de otros para magnificar el poder de una única raza (León Bernardo, 2017, p. 23).

\section{EN CUBA}

La discriminación racial hacia el negro en Cuba, tiene como antecedente la práctica, por más de cuatro siglos, primero por el gobierno colonial español y continuada luego por el sistema neocolonial impuesto tras la primera intervención norteamericana, que representó la negación del ideal martiano de una República con todos y para el bien de todos. (Castro-Monterrey, 2012, p. 363).

El racismo funciona en ambos sentidos fundamentalmente: los discriminados que pueden también asumir un esquema discriminatorio y la persona racista que no se reconoce como tal pero tiene marcadamente establecido la diferencia y lo expresa abiertamente o de forma encubierta y otros que la emplean como elementos costumbristas. (Jiménez y Arroix, 2017, p. 260).

El racismo es una construcción sociocultural del capitalismo, esencialmente histórica. Sus credos, fetiches y estereotipos se encargaron de racializar las nuevas relaciones de producción para garantizar la acumulación originaria de capital, con máximo abaratamiento de la fuerza de trabajo y coherencia formal con la prédica homogenizadora del cristianismo. (Romay, 2014, pp. 50-51).

La investigadora Dra. Gisela Arandia "dice que negar el racismo es una manera ingenua de perpetuarlo". (Blog negra cubana tenía que ser, 2016).

Las opiniones anteriores tienen como fundamentación general, que el objetivo de las clases dominante fomentó el racismo, para dividir, categorizar e identificar. Fue una estrategia fundamentada en la explotación del hombre por el hombre por la supremacía poderosa, impuesta en la sociedad. Cuyo objetivo económico, político y social, respondía a este proceder.

Así las cosas que pasan en la actualidad, se remontan a una cadena de subordinación histórica, de las clases dominantes a las más pobres. Por eso, surge la interrogante, de cómo se resolverán cosas tan acuciantes, si hay algunos/as que están estáticos/as, y no les interesa que opinión pública se mueve respecto a este tema? Y la respuesta está, en la desarticulación de todo ese andamiaje construido; en la sistematización de saberes, en la discusión teórica de una memoria histórica documental por voces coherentes con el discurso pasado y actual del tema racial. 


\section{CASAS COMUNITARIAS Y TALLERES DE TRANSFORMACIÓN INTEGRAL DE LOS BARRIOS (TTIB)}

Amplios sectores de nuestra población se han concientizado de la problemática racial que aun enfrentamos, tanto individual como colectivamente, como los que formamos parte de la Cofradía de la Negritud, ARAAC o la Comisión Aponte. Todo lo mencionado releva la existencia de elementos que accionan de manera objetiva y crean una atmosfera subjetiva muy positiva, retroalimentándose de las experiencias que nos llegan de los hermanos que luchan como nosotros en otras latitudes de nuestro continente y de la expansión de nuestra lucha a través de las casas comunitarias. Éstas están llamadas a convertirse en núcleos principales en esa lucha por las acciones -que por otro lado ya realizan- con el objetivo de difundir aspectos históricos, sociales y culturales, silenciados o abordados muy superficialmente por nuestra historiografía, tanto como el denunciar las manifestaciones discriminatorias y exigir una legislación que las penalice. (Fernández, 2015, p. 104).

En relación con lo expuesto por Fernández, se puede afirmar, que La Casa Comunitaria Paulo Freire, es ejemplo de acciones efectuadas en la Comunidad de La Ceiba, Balcón Arimao, La Habana, en resaltar y divulgar la historia, cultura, tradición e identidad; fue una de las participantes, junto a otras instituciones y organizaciones, en coordinación con la Universidade do Estado do Amazonas, en colaborar en el boletín informativo

Iroko, El espíritu de lo sagrado. Identidad de la Comunidad de La Ceiba, Balcón Arimao, La Habana; cartografía social que recoge las visiones, los testimonios de lo que nosotros como pobladores de la comunidad reconocemos como elementos que son, que forman parte importante de ella, las casas templos, gremios artesanales, artistas, santeros y religiosos, líderes y lideresas mayores, testimonios. Hay testimonios muy, muy sentidos, que tuvimos la oportunidad de ver hoy, historias que forman parte ya de lo imaginario, de la cultura local, del imaginario popular y que son importantes, porque son matrices que conforman la identidad de las personas, de nosotros, el hombre y le dan un sentido a nuestro barrio. (Sánchez y Galves, 2016, p.13).

Sin embargo, no es solamente las casas comunitarias las que deben actuar o luchar, también están los proyectos comunitarios e instituciones, que radican en esas comunidades; y es entre todos, que poco a poco la comunidad debe dar pasos positivos en resolver y trabajar en esos problemas sociales.

Cada uno de ellos, es un eslabón en esa cadena sistémica de la sociedad cubana. Juegan un papel protagónico en acciones a nivel de base, que pueden impulsar a cambios positivos en los habitantes de esas comunidades.

Por eso,

En 1988 el Grupo para Desarrollo Integral de la Capital (GDIC) propone crear de forma experimental, los Talleres de Transformación Integral de los Barrios en tres barrios de la ciudad con deterioro físico-social, dos en la zona central (Cayo Hueso y Atarés) y otro en la periferia sur (La Guinera). Ellos tenían la orientación de realizar un trabajo donde se reflejaran los componentes principales, el social y el físico en estrecha relación, que aglutinara el elemento político-ideológico, que permitiera una valoración de las acciones entre los vecinos y las organizaciones de la zona, con la idea de que si resultaba positiva, se extendiera a otros barrios de la Capital. (Barbón, M.R., 2010, p. 31). 
Los talleres surgieron como pequeños equipos multidisciplinarios, integrados por arquitectos, sociólogos, sicólogos, trabajadores sociales, técnicos en construcción. Todos profesionales afines al trabajo social y constructivo.

El objeto social de los TTIB es promover la integración de las instituciones y otros actores que actúan en la comunidad para la transformación integral y el mejoramiento de la calidad de vida de sus habitantes, a través de un proceso de planeamiento participativo, que propicie además la coordinación de las acciones necesarias para su materialización. (Alemán, 2009, p. 56).

En la actualidad se ha incrementado el número de comunidades que cuentan con estos talleres. Sus líderes promueven, divulgan actividades, intercambian experiencias, capacitan, propician peñas culturales; y desempolvan personalidades históricas de esas comunidades, que ayudan a los nativos a comprender más sobre su historia, identidades, tradiciones, cultura y de las luchas en el pasado y presente en contra de la discriminación y el racismo.

No cabe dudas, que la actuación de las casas comunitarias y los TTIB, en las comunidades, marco un antes y un después. En el momento que surgieron fue una salida para trabajar en función de impulsar el mejoramiento de los barrios y profundizar en las acciones inmediatas que necesita la comunidad; pero ahora, las cosas siguen andando, y la dinámica de la vida, da riendas a mayor creatividad, innovación y conocimiento. Los habitantes actuales están idenficados con un contexto nacional e internacional diferente. Por eso el trabajo en equipo es fundamental. Hacer uso del lenguaje oral y escrito, requiere de una mayor profundización en los estudios e investigaciones. De una mayor articulación entre diferentes actores a nivel micro y macro, desde cualesquier escenario que se pueda.

Mirar y respirar los cambios, da fortaleza interior. Es momento de unir y sumar, aunque parezca que la suma es poca; la última palabra aún falta por ser dada y el trabajo a nivel de comunidad todavía demanda de más sensibilidad, participación y confrontación entre todos y todas.

\section{EMPODERAMIENTO EN AMBIENTE DIGITAL DE LAS CASAS COMUNITARIAS, PROYECTOS COMUNITARIOS QUE TRABAJAN EL TEMA RACIAL}

Actualmente es importante tanto empoderarse en el ambiente físico, como en el ambiente digital. Muchas de las coordinadores/as de proyectos comunitarios y casas comunitarias, están consciente de la necesidad de actuar también en estos espacios; pero las infraestructuras, condiciones económicas y poco conocimiento de cómo dar los primeros pasos y que hacer, han frenado inundar las redes sociales en internet, de todo el arduo trabajo que se realiza en ambiente físico con los diferentes actores locales.

Poco a poco ha ido floreciendo la idea de llegar a estas fronteras virtuales. De encontrarse con otros/as activistas, coordinadores/as o actores/as, que habitan en las redes sociales, y desconocen de las similitudes y diferencias que hay entre un contexto y otro. Saber socializar e intercambiar, es la construcción de conocimiento, desde las experiencias vividas; es la confrontación de saberes de sujeto a sujeto, con un análisis crítico y constructivo.

El trabajo que se realiza día a día, en las calles, barrios, comunidades, instituciones, organizaciones tradicionales, deben ser visibilizados/as en las redes sociales (en internet) 
y enriquecidas con el aporte de todos los que hacen contribuciones creativas a la narrativa transmedia, siendo estas personas prosumidores/as y procreativos/as. La educación popular en el entorno digital enriquece la interacción comunicativa.

La realidad no es estática, es dinámica, cambiante y con nuevas barreras a derribar. Hombres y mujeres cada día deben adaptarse a los nuevos cambios positivos o negativos, que modifican la vida cotidiana, con saberes que antes eran ajenos, en un contexto determinado; pero sin embargo ahora hacen cambiar la dinámica de la vida, y han llegado para quedarse e insertarse en las rutinas diarias.

"El ser humano no se concibe dentro de una esfera cerrada de interioridad estática; continuamente tiene que externalizarse en actividad". (Berger y Luckmann, 2006, p. 71).

La bioquímica de la vida es enriquecedora. Las formulas pueden ser modificadas o adaptadas al entorno social que se está viviendo; pero sin perder la visión, ni el sentido, que se parte de un antecedente fuerte, que no se puede olvidar, y que ayuda a solventar respuestas para derribar muros invisibles y visibles, y ser parte de la participación popular en ambiente digital, por un bien común.

Como dice Roselló,

la participación y el diálogo no son posibles por decretos, mucho menos pueden medirse por porcentajes de asistencia a una actividad o movilización. Son procesos interrelacionados, esenciales para la construcción colectiva y la socialización del poder (pensar creativamente, decir con honestidad, hacer para transformar, decidir con justeza...), tal como lo anuncia el socialismo. (Roselló, Lezcano y Menéndez, 2017, p. 98).

"La comunicación no es un eje que camina solo, es parte sustantiva del entramado que se construye entre la gente, porque favorece las relaciones humanas y el intercambio de información, de conocimientos, de experiencias". (Roselló, Lezcano y Menéndez, 2017, p. 102).

Este paradigma nos habla de aprovechar disímiles vías y medios para comunicarnos, desde la riqueza de las manifestaciones artísticas, el poder de la cultura popular, la efectividad del intercambio cara a cara, las tecnologías de información y comunicación digitales. Eso sí, no se comunica en abstracto ni a una masa homogénea o manipulable, por el contrario, se dialoga con audiencias específicas, con demandas y realidades puntuales, con una historia y un presente, que condiciona su comprensión de los fenómenos. (Roselló, Lezcano y Menéndez, 2017, pp. 104-105).

El profesor estadounidense Henry Jenkins considera que en los medios la relación entre productores y público está cambiando, pues no solo aparecen figuras como la del prosumidor, sino que los fans generan más presión y ahora se involucran en la circulación de los contenidos, de manera que la participación en estos momentos es la clave en la economía de los medios. (Jenkins, 2014).

Garantizar el desarrollo de capacidades en el ambiente digital también debe ser una visión de hacia donde los actores que trabajan las temáticas raciales deben enfocarse. Debe ser una fortaleza tener esta proyección. Cada vez es importante tener identificado a esos usuarios e internautas en las redes sociales que confluyen en la lucha contra la discriminación, y a su vez, del movimiento social que se vive en ambos espacios. Escuchar e intercambiar, puntos de vistas, es una manera de pensar a favor de edificar 
puentes de solidaridad y unidad, derribando fronteras virtuales y físicas; es saber aprovechar las oportunidades que están presentes en la contemporaneidad, en entorno tradicional y el entorno virtual.

Estas plataformas, que están concebidas bajo un nuevo diseño visual, de interacción, estimulan la presencia de los usuarios, su interrelación con otros, hasta convertirse en una especie de "plaza pública" cuya existencia misma depende en primer lugar de los usuarios, de los contenidos que ellos compartan y las relaciones que establezcan. Es por ello, y dada su magnitud, que los medios de comunicación, las empresas y muchas otras organizaciones han buscado estar también presentes en este escenario, como forma de acercarse a sus audiencias. Como nunca antes hoy sabemos dónde encontrar a los usuarios, pero como nunca antes también estas instituciones deben considerar el diálogo como fórmula en un espacio donde el individuo tiene, se podría decir, el poder. (Pérez, 2015. p. 31).

También se hace preciso tomar en cuenta que las plataformas de mayor éxito, tomando como centro la cantidad de usuarios que han logrado reclutar alrededor de ellas, han surgido y se han desarrollado en los países de mayor poder económico, fundamentalmente Estados Unidos. Es este un dato sumamente importante que, más allá de evidenciar la indiscutible situación ventajosa que ello implica para este país, es también muestra de la desigualdad que conlleva para otros países el hecho de participar "activamente" en una red comunicativa en donde otros imponen sus reglas del juego. Lo que no significa que estas puedan ser modificadas hasta un punto, usadas para beneficio propio o bien buscar maneras de crear nuestras propias plataformas. (Pérez, 2015, p. 31).

"En este sentido es conveniente reflexionar acerca de que la tecnología no es desligable del contexto social, económico y político y a partir de esto se entiende que ella es un instrumento puesto al servicio de este contexto". (Casa, 2005, p. 7).

Atender lo que aborda Casa, es diseñar estrategias flexibles y diferentes, que respondan a equipos de trabajo interconectados, comunicados, innovadores y creativos, con una responsabilidad social, en correspondencia al contexto global actual.

En Cuba hace algunos años ya es visible, en algunos blogs como Negra cubana tenía que ser; afrocubaweb; daisyrubieracastillo; afrofeminas; estebanmoralesdominguez.blogspot; y un directorio de afrocubanas con un índice alfabético donde se puede recuperar por especialidades y profesiones tales como: académicas, diplomáticas, bibliotecarias, consultoras, activistas, actrices y otras tantas profesiones. Estos sitios existentes en las redes sociales de internet, realizan un serio trabajo de divulgación, promoción e investigación, donde se visibiliza estereotipos positivos sobre las mujeres negras cubanas afrodescendientes, se denuncia, y se representa concretamente todo ese potencial humano que genera y trabaja en función del desarrollo de Cuba; y sus conocimientos lo ponen en función de responder al contexto actual, que les ha tocado vivir.

\section{MEDIOS DE COMUNICACIÓN MASIVA}

La todavía escasa visibilidad en los medios de comunicación social cubanos de las problemáticas que se analizan en este ensayo tampoco contribuye a fomentar conciencia sobre la urgencia de resolver, con la contribución de todos, las tensiones interraciales latentes. Aunque el programa de corte informativo Mesa redonda comenzó a estimular el debate desde la televisión en enero de 2010 y, 
posteriormente, intelectuales y artistas de alto reconocimiento social -como Carlos Acosta y Gerardo Alfonso- aportaron valiosos testimonios y argumentos en sendas comparecencias en el espacio televisivo Con dos que se quieran, nuestros medios acusan aún inhibiciones temporáneas, rémora de la época en que los dramatizados, el periodismo de opinión y los programas de participación sobre 'temas negros' se consideraban perturbadores e inoportunos." (Romay, 2014, pp. 117-118).

Se distingue la importancia que tiene incluir el tema en los diferentes medios. Es acertada la visibilidad en escenarios como este, por la facilidad de llegar a todo tipo de público, en un corto margen de tiempo.

En el Plan de Acción del Programa del País, en la Parte IV, Programa Propuesto, Los medios de comunicación se constituyen en un ámbito estratégico para promover juicios de valor, representaciones sociales y prácticas participativas y no discriminatorias. Capacitar a los decisores y decisoras, a comunicadores y comunicadoras, a estudiantes de periodismo, comunicación y diseño para una comunicación desde la perspectiva de género potenciaría la cultura de equidad necesaria para impulsar verdaderas transformaciones en la situación y condición de mujeres y hombres. (Gobierno de la República de Cuba- UNFPA, 2013, p. 9).

La realidad es una construcción social que a su vez permite y construye a la sociedad. Por tanto los medios tienen la función de crear esa realidad construyéndola mediante la observación de la sociedad y difundiéndola en ella. Cuanto más compleja y grande es la sociedad, tanto más importante es la función de los medios, pues sin ellos una sociedad de dimensiones y complejidad más grandes no podría funcionar como sociedad (Watzlawick y Krieg, 1994, p. 125).

Los medios son culpables de las subjetividades negativas o positivas, que cada persona va creando en su realidad. Esa información visual, de ese televidente, se alimenta y va creciendo. Esa población, va idealizando estereotipos.

La realidad que creo para mí como observador es mi realidad de primer orden. Al informar sobre ella, creo una realidad de segundo orden, o sea, 'un mapa del mapa' que luego se convierte a su vez en una realidad de primer orden para los espectadores que no fueron testigos de acontecimientos informados. (Watzlawick y Krieg, 1994, p. 130).

Es la realidad del otro. Es la noticia elaborada y puesta a disposición de la población. Es la subjetividad del otro, presentada en un producto comunicativo que transmite algo, de una manera, que el público va a identificar como su realidad.

En una entrevista realizada por Anette Jiménez Marata a la Dra. María del Carmen Zabala, profesora titular de la Facultad Latinoamericana de Ciencias Sociales (FLACSOCuba), acota la especialista, considero que la dimensión de la desigualdad menos reflejada y analizada en la TV es la racial, por la limitada presencia de personas negras y mestizas en los programas de televisión y porque no se presentan ni problematizan las inequidades existentes según la variable racial, vinculadas a desventajas de diferente orden (Jiménez, 2017). 
"El diálogo e intercambio constante y fluido entre académicos y realizadores audiovisuales es un recurso indispensable para lograr una representación profunda, verosímil y orgánica de estos temas tan sensibles para la sociedad". (Jiménez, 2017).

El punto de vista de la problemática racial que tienen los especialistas de los medios, es importante conocerlo; porque la elaboración de una noticia o producto comunicativo, estaría en juego por aquellos que no estudian y no están sensibilizados, sobre ese tópico. Se estaría construyendo una realidad alejada del contexto social cubano.

Es primordial la responsabilidad social que se debe tener para representar, la realidad de algo. Toda acción que se realiza en torno a crear estereotipos positivos o negativos conduce a consecuencias en la sociedad.

\section{CONCLUSIONES}

El análisis documental de elementos teóricos-conceptuales permitió reflejar en el presente trabajo; como diferentes autores, de diferentes regiones, han logrado visibilizar la problemática racial.

En la actualidad, es importante profundizar en acciones puntuales, para eliminar o contrarrestar las construcciones sociales e imaginarios populares, que se han ido arrastrando desde la colonización, hasta el presente.

Representar de forma adecuada la información real y pertinente de negros/as en los censos de población, en los medios de comunicación masiva, en la producción académica, cultural y social; propicia acabar con los estereotipos racistas en la población, y es estar en sintonía con un desarrollo social acorde al contexto actual.

\section{BIBLIOGRAFÍA}

Alemán Díaz, M. (2009). Mil palabras, una imagen: un acercamiento a la imagen pública del Taller de Transformación Integral del Barrio de la Comunidad de Micro $X$ del Consejo Popular Alamar Este (Tesis de diploma). La Habana: Universidad de la Habana, Facultad de Comunicación.

Almeida Junco, Y. (2010). Racismo: Un mal que ronda la sociedad contemporánea. Una reflexión desde Cuba. Serviço Social \& Realidade, Franca, XIX(1).

Álvarez-Buylla, C. C. (2014). Desde la audacia, descontruyendo mitos y configurando realidades (Tesis de diploma). La Habana: Universidad de la Habana, Facultad de Comunicación.

Baldwin, J. (1961). Nadie sabe mi nombre (Titivillus). Recuperado de www.espapdf.com

Berger, P. L. y Luckmann, T. (2006). La construcción social de la realidad. Buenos Aires: Amorrortu.

Bourdieu, P. (2000). La dominación masculina. Barcelona: Anagrama.

Carbonell, W. (2005). Cómo surgió la cultura nacional. 2da. Edición corregida. La Habana: Biblioteca Nacional José Martí. 
Casa Tirao, B. (2005). Información, Tecnología e Industria. En Morales Campos, Estela y Ríos Ortega, Jaime (coords.) Mesa Redonda. Ética e Información: memoria, 24, 25 de septiembre del 2003. México: UNAM, Centro Universitario de Investigaciones Bibliotecológicas

Casanova García, M. A. (2014). Debate racial: entre dichos y hechos. El debate público intelectual en torno a la problemática racial durante 2008-2013 (Tesis de diploma). La Habana: Universidad de La Habana, Facultad de Comunicación.

Castro-Monterrey, P. M. (2012). El negro en el contexto social del primer decenio del siglo XX en Santiago de Cuba. Revista Santiago, 128.

Colino, C. (2009). Racismo, en Reyes, Román (Dir.) Diccionario crítico de Ciencias Sociales. Recuperado de http://webs.ucm.es/info/eurotheo/diccionario/R/racismo.htm

Definición de racismo (2015). Recuperado de https://conceptodefinicion.de/racismo/

De la Iglesia, M. (2002). Representaciones acerca de la práctica del comunicador. Material de la Cátedra Ferrarós de la Universidad de Buenos Aires (Argentina). Recuperado de http://www.catedras.fsoc.uba.ar/ferraros/BD/mdi\%20representaciones\%20cc.pdf

Duncan, Q. (s.f.). Documento I. Génesis y evolución del racismo real-doctrinario. Recuperado de https://www.encaribe.org/Files/Personalidades/quince-duncanmoodie/texto/Duncan.pdf

Fernández Robaina, T. (2015). Los términos afrocubano y afrodescendiente y la importancia de ARAAC para Cuba, en: Identidades políticas en tiempos de Afrodescendencias: Auto- Identificación, Ancestralidad, Visibilidad y Derecho, (pp. 91125). Ciudad Autónoma de Buenos Aires: Corregidor.

Gobierno de la República de Cuba- UNFPA. Plan de Acción de Programa del País 20142018. (Versión borrador 2013). Cuba: Gobierno de la República de Cuba y el Fondo de Población de las Naciones Unidas - UNFPA.

Grosfoguel, R. (2006). Actualidad del pensamiento de Césaire: redefinición del sistemamundo y producción de utopía desde la diferencia colonial. En Césaire, Aimé. Discurso sobre el colonialismo. Madrid: Ediciones Akal, S. A.

Inter Press Service en Cuba (IPS) (2016). Negar el racismo es una manera ingenua de perpetuarlo", dice investigadora cubana. Recuperado de

http://www.ipscuba.net/sociedad/negar-el-racismo-es-una-manera-ingenua-deperpetuarlo-dice-investigadora-cubana/

Jenkins, H. (2014). El prosumidor y la cultura participativa. Recuperado de http://lecturalab.org/story/Henry-Jenkins-el-prosumidor-y-la-cultura-participativa 4664

Jiménez Marata, A. (2017). Las dimensiones de la desigualdad en la pantalla. Recuperado de http://www.tvcubana.icrt.cu/seccion-temas/2893-las-dimensiones-dela-desigualdad-en-la-pantalla 
Jiménez Vega, M. P. y Arroix Jiménez, T. (2017). Reflexiones sobre las manifestaciones de racismo en Cuba desde 1959. Universidad \&Ciencia, 6(Especial UNICA).

Malcom X (1991). Malcolm X. Vida y voz de un hombre negro. Autobiografía y selección de discursos. Navarra: Txalaparta Editorial.

Martínez, B. (2014). Territorios de identificación: una poética de la resistencia de la gente en Turbo (Tesis de Maestría). Universidad de Antioquia. Recuperado de http//ayura.udea.edu.co:8080/jspui/bitstream/123456789/618/1/LB0247.pdf

Mendiazábal, N. (2007). Los componentes del diseño flexible en la investigación cualitativa. En Vasilachis de Galindo, Irene (Coord.), Estrategias de investigación cualitativa, (pp. 65-106). Buenos Aires, Argentina: Gedisa.

Negra Cubana Tenía que ser. Recuperado de https://negracubanateniaqueser.com/2016/12/08/sabado-del-libro-emergiendo-delsilencio-mujeres-negras-en-la-historia-de-cubal

Oquendo Barrios, L. (2006). Cimarronaje y antirracismo. La Habana: Biblioteca Nacional José Martí, Eds. Bachiller.

Pérez Alonso, B. (2015). ¿Mi perfil soy yo? Acercamiento a las concepciones sobre la construcción de identidad en los servicios de redes sociales en internet (tesis de Maestría). La Habana: Universidad de La Habana, Facultad de Comunicación.

Quijano, A. (2017). ¡Qué tal raza!, en Campoalegre Septien, Rosa y Bidaseca, Karina (Eds.) Más allá del decenio de los pueblos Afrodescendientes. Ciudad Autónoma de Buenos Aires: CLACSO.

Rojo, G. (2009). Globalización e identidades nacionales y postnacionales... ¿de qué estamos hablando? La Habana: Fondo Editorial de la Casa de las Américas.

Romay Guerra, Z. (2014). Elogio de la altea o las paradojas de la racialidad. La Habana: Fondo Editorial Casa de las Américas.

Roselló Reina, T.; Lezcano Lavandera, Y. y Menéndez García, Y. (2017). Comunicación popular en Cuba: desafío de pronunciar el socialismo, en Portal Moreno, Rayza; Garcés Corra, Raúl y Pedroso Aguiar, Willy (comps.) Información, comunicación y cambio de mentalidad. La Habana: Editorial de Ciencias Sociales.

Sánchez Saavedra, A. y Galves Sánchez, L. (2016). La Cartografía de La Ceiba, en Iroko, El espíritu de lo sagrado. Identidad de la Comunidad de La Ceiba, Balcón Arimao. La Habana: Manaus: UEA.

Stuart, H. (2013). Discurso y Poder. Restrepo, E. (Presentación) y Soto Sulca, R. (Edit.) Huancayo, Perú: MELGRAPHIC E.I.R.L.

Watzlawick, P. y Krieg, P. (1994). El ojo del observador. Contribuciones al constructivismo. Barcelona: Gedisa. 